Check for updates

Cite this: Nanoscale Adv., 2019, 1, 4393

\title{
Threshold reduction and yield improvement of semiconductor nanowire lasers via processing- related end-facet optimization $\uparrow$
}

\author{
Juan Arturo Alanis, (D) a Qian Chen, (D) ${ }^{b}$ Mykhaylo Lysevych, (D) ${ }^{c}$ Tim Burgess, ${ }^{d} \mathrm{Li} \mathrm{Li}^{\mathrm{c}}$ \\ Zhu Liu, (D) ${ }^{b}$ Hark Hoe Tan, (D) ${ }^{d}$ Chennupati Jagadish (D) ${ }^{d}$ and Patrick Parkinson (DD *a
}

\begin{abstract}
Both gain medium design and cavity geometry are known to be important for low threshold operation of semiconductor nanowire lasers. For many applications nanowire lasers need to be transferred from the growth substrate to a low-index substrate; however, the impact of the transfer process on optoelectronic performance has not been studied. Ultrasound, PDMS-assisted and mechanical rubbing are the most commonly used methods for nanowire transfer; each method may cause changes in the fracture point of the nanowire which can potentially affect both length and end-face mirror quality. Here we report on four common approaches for nanowire transfer. Our results show that brief ultrasound and PDMS-assisted transfer lead to optimized optoelectronic performance, as confirmed by ensemble median lasing threshold values of 98 and $104 \mu \mathrm{J} \mathrm{cm}^{-2}$ respectively, with nanowires transferred by ultrasound giving a high lasing yield of $72 \%$. The mean threshold difference between samples is shown to be statistically significant: while a significant difference in mean length from different transfer methods is seen, it is shown by SEM that end-facet quality is also affected and plays an important role on threshold gain for this nanowire architecture.
\end{abstract}

\section{Introduction}

Nanowires have been widely studied as coherent light sources for potential integration in the field of on-chip nanophotonics. ${ }^{1-4}$ Due to their characteristic elongated shape and reflective end facets, nanowires can act as a Fabry-Pérot resonant cavity under excitation. ${ }^{5}$ Recent progress has led to an increase in efficacy of semiconductor nanowire lasers through both improved nanowire geometry and optimized active region absorption. ${ }^{6-14}$ In many applications it is necessary to transfer the wires to a structured ${ }^{15}$ or lower index substrate such as silica aerogel, ${ }^{16}$ indium tin oxide (ITO) ${ }^{7}$ or $\mathrm{SiO}_{2},{ }^{17}$ and, while many efforts have contributed to the improvement of lasing operation, the impact of different nanowire transfer techniques on nanowire geometry and, consequently optical performance has so far not been addressed.

\footnotetext{
${ }^{a}$ Department of Physics and Astronomy and the Photon Science Institute, The University of Manchester, Manchester, UK. E-mail: patrick.parkinson@manchester.ac.uk

${ }^{b}$ Department of Materials, The University of Manchester, Manchester, UK

${ }^{c}$ Australian National Fabrication Facility, The Australian National University, Canberra, Australia

${ }^{d}$ Department of Electronic Materials Engineering, Research School of Physics and Engineering, The Australian National University, Canberra, Australia

$\dagger$ Electronic supplementary information (ESI) available: Summary of length measurements by SEM. SEM images of pseudo-nanowires. Details on the ANOVA test and summary of calculations and results. See DOI: $10.1039 / \mathrm{c} 9 \mathrm{na} 00479 \mathrm{c}$
}

Understanding the impact of processing steps on single nanowire lasers cannot be achieved through single or few wire measurements, as any systematic variation is masked by a high degree of wire-to-wire heterogeneity common to this architecture. Here we report on four of the most commonly used methods to transfer nanowires by investigating their effect on both geometry and optical performance using hundreds of single nanowire spectroscopic measurements for each method. ${ }^{18,19}$ Using automated data acquisition techniques combined with a robust statistical analysis is essential to identify correlations that would otherwise be impossible to resolve from a smaller set of measurements. ${ }^{20}$ Transfer methods used included a solution-based transfer by ultrasonication for $5 \mathrm{~s}$ and $100 \mathrm{~s}$, a dry transfer by rubbing and PDMS stamping onto $z$-cut quartz substrates. The nanowires used for this study are a p-doped GaAs core-only architecture grown by Au-assisted vapor liquid solid mechanism as reported by Burgess et al. , $^{8,19}$ We compare the impact of transfer method on nanowire length, lasing yield (defined as the fraction of nanowires tested which display lasing behaviour) and lasing threshold, and correlate it with end-facet quality imaged using Scanning Electron Microscopy (SEM). We find that nanowires from sample transferred by $5 \mathrm{~s}$ ultrasound have the best lasing performance, with a median lasing threshold of $98 \mu \mathrm{J} \mathrm{cm} \mathrm{cm}^{-2}$ and a lasing yield of $\sim 72 \%$, followed by PDMS with $104 \mu \mathrm{J} \mathrm{cm}^{-2}$ and a reduced lasing yield of $\sim 60 \%$. Additionally, we observe a statistically significant difference in mean nanowire length between the 
ultrasound and PDMS transferred samples of $2.68 \pm 0.8 \mu \mathrm{m}$ and $2.00 \pm 0.65 \mu \mathrm{m}$ respectively. While threshold gain is expected to have an inverse relationship with cavity length, this is not clearly observed here; the mean lasing threshold from nanowires transferred by PDMS is in fact comparable to $5 \mathrm{~s}$ ultrasonication, which we attribute to an improvement in end-facet quality in PDMS transfer as observed in SEM imaging. Nanowires transferred by rubbing or $100 \mathrm{~s}$ ultrasound suffer from lower optoelectronic performance due to poor end-facet quality or higher morphological degradation when exposed to a longer ultrasonication time.

\section{Results and discussion}

Four samples were prepared using nanowires originating from the same part of the same growth wafer: NW-US5, NW-US100, NW-R and NW-PDMS. Samples NW-US5 and NW-US100 were prepared by placing the growth substrate into iso-propylalcohol and dispersing nanowires into solution using a camSonix C575/ T ultrasound bath (which uses a standard sine-wave modulation of $37 \mathrm{kHz}$ and has a peak power of $600 \mathrm{~W}$ ) for 5 and 100 seconds respectively, before drop depositing the solution onto clean quartz substrates. Sample NW-R was prepared by gently rubbing the as-grown nanowires directly onto quartz substrates, transferring wires using micro-mechanical cleavage. Sample NWPDMS was prepared by using a cured PDMS stamp to peel nanowires from their growth substrate using gentle pressing. The nanowire-laden stamp was then pressed onto a quartz receiver substrate to adhere them. SEM images of free-standing nanowires on their growth substrate prior to being transferred are shown in the ESI. Since transferred nanowire densities were observed to vary between $200-1000 \mathrm{NWs} \mathrm{mm}^{-2}$, we randomly selected and studied 300-400 isolated nanowires from each sample to avoid overlapping wires.

Nanowire length for each sample was measured by optical microscopy imaging; an example image of a randomly selected nanowire from sample NW-US5 is shown in the inset of Fig. 1a. Additionally, we studied lasing performance by power dependent photoluminescence at room temperature, using a $620 \mathrm{~nm}$ pulsed laser at a repetition rate of $250 \mathrm{kHz}$, and a $170 \mathrm{fs}$ pulse duration. The beam was de-focused to a circular spot size of $\sim 360 \mu \mathrm{m}^{2}$ to ensure uniform excitation along the whole nanowire. To avoid excitation of multiple nanowire lasers, we selected isolated nanowires with a minimum separation of at least $20 \mu \mathrm{m}$. Fig. 1a shows the emission image above lasing threshold (and the brightfield image, inset). The corresponding power dependent spectra from the same nanowire is shown in Fig. 1b. For comparison, a high-resolution optical image of another wire taken at higher magnification is shown in Fig. 1c. At low excitation energy a broad emission centred at $\sim 860 \mathrm{~nm}$ is observed. As the excitation fluence increases to $\sim 50 \mu \mathrm{J} \mathrm{cm}^{-2}$ an initially narrow peak at $856 \mathrm{~nm}$ starts to emerge and continues to grow in intensity with excitation above threshold. The appearance of a secondary peak at $826 \mathrm{~nm}$, associated with an additional longitudinal Fabry-Pérot cavity mode is observed at a fluence of $\sim 125 \mu \mathrm{J} \mathrm{cm}^{-2}$. A blue shift in the main and second lasing peak is observed as a function of fluence, from 856 to
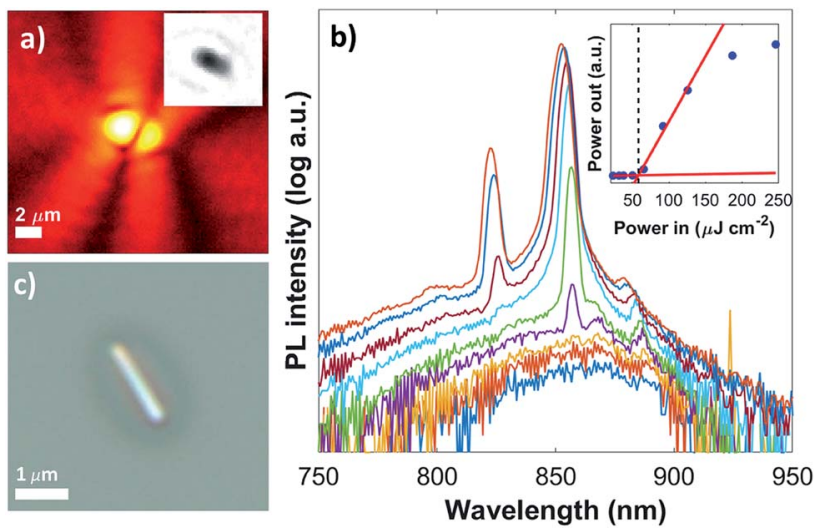

Fig. 1 (a) Lasing emission image of a single nanowire from the US5 method under pulsed excitation; the inset shows the bright-field optical image used to estimate the length by machine vision on the same scale, and (b) corresponding power dependent emission spectra from the lasing emission. A light-in versus light-out plot is shown in the inset, where a linear fit is used for the spontaneous and stimulated emission regimes, and the calculated lasing threshold is indicated by a dashed line. (c) High-resolution optical image of a different nanowire prepared using the same method.

$852 \mathrm{~nm}$ and 825 to $822 \mathrm{~nm}$ respectively. This blue shift is associated to the change in material refractive index induced by the increased photo-excited carrier density, as previously reported for GaAs. ${ }^{\mathbf{6 , 8 2 1 - 2 3}}$ At high excitation fluence, we observe a lasing peak line-width of $\sim 10 \mathrm{~nm}$, which is larger than that seen just above threshold (and larger than typically observed for nanowire lasers). We attribute this large line-width to a smearing effect caused by the carrier density (and hence effective refractive index) varying during the laser emission. ${ }^{24}$ The lightin $v s$. light-out (LILO) plot is shown in the inset of Fig. 1, where a linear fit was used for the spontaneous emission and stimulated emission regimes, and the lasing threshold was taken as the intersection of the two lines as previously described $^{18}\left(58 \mu \mathrm{J} \mathrm{cm}^{-2}\right.$ for this wire).

Fig. 2a shows the length histograms corresponding to each sample, where a normal probability distribution function is used to fit the data. Samples NW-US5 and NW-US100 had the longest average length with values of $2.68 \pm 0.8 \mu \mathrm{m}$ and $2.44 \pm$ $0.65 \mu \mathrm{m}$ respectively, whereas samples NW-PDMS and NW-R have a mean length of $2.00 \pm 0.65 \mu \mathrm{m}$ and $1.97 \pm 0.65 \mu \mathrm{m}$ respectively. This result indicates that nanowires transferred by solution-based methods seem to fracture closer to the base resulting in longer wires. These values were confirmed by SEM, where we measured 12 and 8 randomly selected nanowires from samples transferred by PDMS and rubbing respectively, and 6 nanowires transferred by ultrasound for both $5 \mathrm{~s}$ and $100 \mathrm{~s}$ (details in the ESI $\dagger$ ).

Fig. $2 \mathrm{~b}$ shows lasing threshold histograms corresponding to each sample where the log-normal probability density function (PDF) is represented by a red line; median values are summarized in Table 1. We found sample NW-US5 to have the lowest median lasing threshold $\left(T_{\mathrm{m}}\right)$ with a value of $98_{-23}^{+40} \mu \mathrm{J} \mathrm{cm} \mathrm{cm}^{-2}$ and a lasing yield of $72 \%$, where the lasing yield is taken as the ratio 


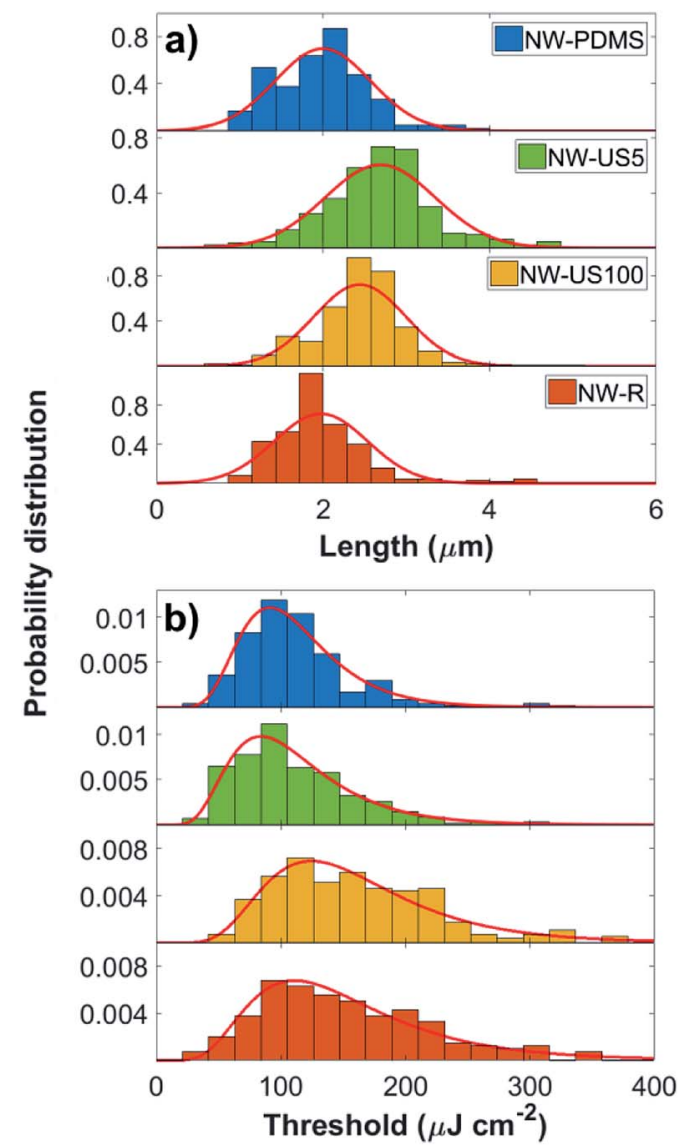

Fig. 2 (a) Length distribution measured from optical imaging and (b) lasing threshold distributions corresponding to each sample. The red line indicates the normal distribution function fit for the length data, and a log-normal distribution fit to the threshold data. A summary of the parameters for the fits in (a) and (b) can be found in Table 1.

between nanowires showing lasing (without undergoing thermal degradation), divided by the total number of wires studied for that sample. Sample NW-PDMS also had a low median threshold of $104_{-21}^{+27} \mu \mathrm{J} \mathrm{cm}^{-2}$; however it had a lower lasing yield of $60 \%$. The lower yield could come from the nature of PDMS stamping where a number of shorter and heavily tapered nanowires are picked up during the transfer process. These pseudo-nanowire structures were observed in SEM imaging on two separate PDMS samples but not found when using any other transfer methods (details in the ESI $\dagger$ ). While samples NW-R and NW-US100 also have high lasing yields (72\% and $76 \%$ ) they showed an increase in median threshold with values of $139_{-38}^{+60}$ and $157_{-46}^{+48} \mu \mathrm{J} \mathrm{cm}^{-2}$ respectively and the largest spread in measured threshold values (Table 1). We note that the mean threshold from sample NW-US5 is higher than the one reported by us in previous works for the same growth. ${ }^{19}$ Given that the samples used for this study were prepared from a different part of the same growth substrate, we attribute this difference to variation in nanowire growth as a function of position.

To verify that the length and threshold difference across all samples is statistically significant we performed an analysis of variance (ANOVA) and Tukey-Kramer tests, ${ }^{25}$ where each sample mean is denoted as $\mu_{\mathrm{PDMS}}, \mu_{\mathrm{R}}, \mu_{\mathrm{US} 5}$ and $\mu_{\mathrm{US} 100}$. ANOVA is used to compare two or more means from independent (unrelated) groups using an F-distribution. The null hypothesis $H_{0}$ for the test is that all the means are equal, in this case

$$
H_{0}: \mu_{\mathrm{PDMS}}=\mu_{\mathrm{R}}=\mu_{\mathrm{US} 5}=\mu_{\mathrm{US} 100}
$$

The alternative hypothesis $H_{1}$ would state that the means are not equal. Therefore, if any of the means are statistically unequal $H_{0}$ is said to be rejected and the test provides a significant result. For our study we ran two ANOVA tests: one on the mean length and another on the mean threshold across all groups. In both cases $H_{0}$ was rejected with a statistically significant evidence at $p \ll 0.01$ which shows a difference in mean length and lasing threshold among the four transfer methods (further details of the one-way ANOVA calculations are given in the ESI $\dagger$ ). To identify which means are statistically different, a Tukey-Kramer (T-K) ad-hoc test was used. Fig. 3a shows the graph of mean length calculated from ANOVA and $\mathrm{T}-\mathrm{K}$ tests. It can be seen that there is a significant difference in mean length obtained by optical microscopy between samples transferred by ultrasonication ( $\mu_{\mathrm{US} 5}$ and $\left.\mu_{\mathrm{US} 100}\right)$ and samples transferred mechanically $\left(\mu_{\mathrm{PDMS}}\right.$ and $\left.\mu_{\mathrm{R}}\right)$. Fig. $3 \mathrm{~b}$ shows the graph of ANOVA and T-K tests for mean threshold, where sample means $\mu_{\mathrm{PDMS}}$ and $\mu_{\mathrm{US} 5}$ show a statistically significant reduction in threshold values when compared with the other methods.

The variation in performance across all samples can be understood from the difference in threshold gain $\left(g_{\text {th }}\right)$. By approximating a nanowire to a cylindrical Fabry-Pérot cavity, ${ }^{26}$ $g_{\text {th }}$ can be expressed as

$$
g_{\text {th }}=\alpha_{0}-\frac{\log \left(R_{\mathrm{Au}} R_{\mathrm{base}}\right)}{2 L},
$$

where $\alpha_{0}$ is the distributive losses $R_{\mathrm{Au} \text {,base }}$ the reflectivity coefficients at the Au tip and base of the nanowire respectively and $L$ is the cavity length. The low mean lasing threshold from sample

Table 1 Summary of lasing yield, threshold (with error bars indicating interquartile range) and mean lengths for each studied transfer technique

\begin{tabular}{lllll}
\hline Method & Yield $(\%)$ & $T_{\mathrm{m}}\left(\mu \mathrm{J} \mathrm{cm}^{-2}\right)$ & Champion $\left(\mu \mathrm{J} \mathrm{cm}{ }^{-2}\right)$ & Mean length $(\mu \mathrm{m})$ \\
\hline NW-US5 & 72 & $98_{-23}^{+40}$ & 38 & $2.68 \pm 0.8$ \\
NW-US100 & 76 & $157_{-46}^{+48}$ & 49 & $2.44 \pm 0.65$ \\
NW-R & 72 & $139_{-38}^{+60}$ & 31 & $1.97 \pm 0.65$ \\
NW-PDMS & 60 & $104_{-21}^{+27}$ & 28 & $2.00 \pm 0.65$
\end{tabular}



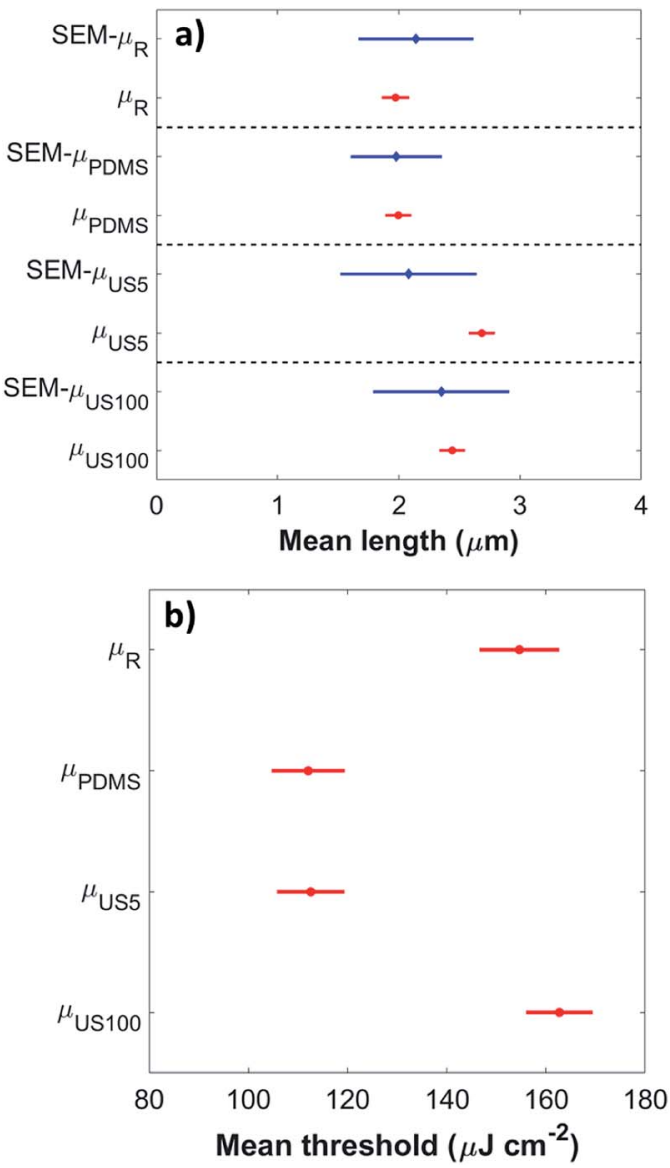

Fig. 3 (a) Mean length calculated from ANOVA and T-K tests, red points correspond to mean length from optical microscopy and blue diamonds correspond to length measured by SEM imaging. (b) Lasing threshold mean values calculated from ANOVA and T-K tests. In both (a) and (b) the bars indicate the standard error corresponding to each group.

NW-US5 is attributed to the increased cavity length whereas sample NW-PDMS, while having length values $\sim 25 \%$ lower but comparable mean threshold, could stem from an improvement in the quality of the nanowire end-facet. Since the Au nanoparticle present in these wires is expected to improve the reflectivity, ${ }^{6}$ and assuming $R_{\mathrm{Au}}$ to be constant across all wires, it is likely that the decrease in threshold from sample NW-PDMS comes from an improvement in facet quality, and therefore $R_{\text {base }}$, at the base of the nanowire. To verify end-facet structure we prepared four samples by transferring nanowires to Si wafers using the methods described above and imaged the base of randomly selected nanowires from each sample by SEM. Fig. 4 shows a difference in end facet quality corresponding to each transfer method. A close inspection of nanowires from samples NW-PDMS show a predominantly flat and smooth surface compared to sample NW-R, which could be contributing to the reduction in threshold gain. An alternative approach to visualise the end facet quality associated with each transfer method is provided by examining the exfoliated growth substrates using SEM after nanowire transfer (data and details in the ESI $\dagger$ ). After transfer, breaking points resulting from PDMS stamping show

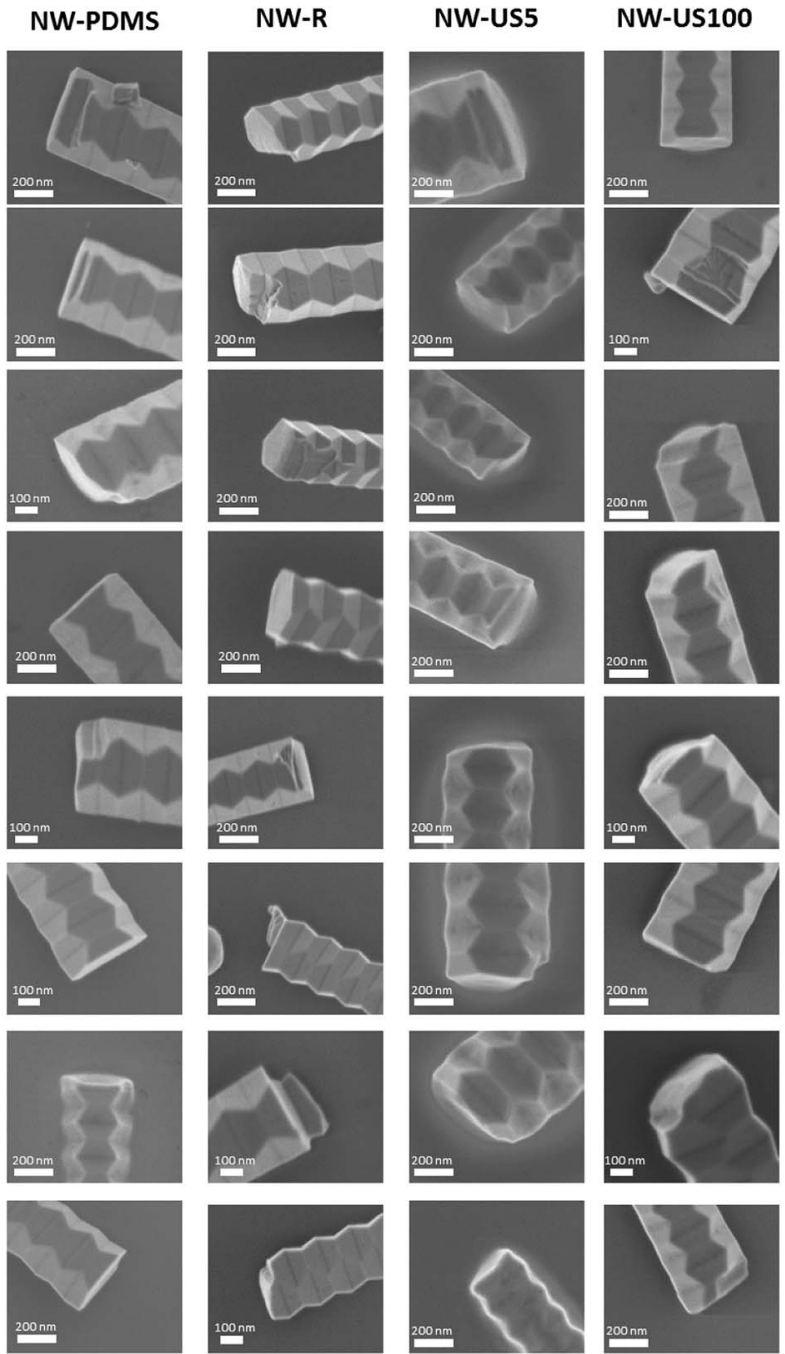

Fig. 4 SEM imagery of the bases of a number of nanowires. Nanowires from sample NW-PDMS show a predominantly smooth and flat fracture point at the base.

predominantly smoother facets when compared with substrates after ultrasound or mechanical rubbing, further supporting the higher cavity quality achievable using the PDMS method. Despite sample NW-US100 having longer lengths, the higher mean threshold suggests that nanowires exposed to longer ultrasonication may be subject to heavier damage. Indeed, crystal dislocation and twinning propagation has been reported for GaAs nanowires under high tensile strain. ${ }^{27}$

\section{Conclusion}

In conclusion, we report optoelectronic performance of p-doped GaAs nanowire lasers transferred by four commonly used methods: mechanical rubbing, PDMS stamping and ultrasound for 5 and $100 \mathrm{~s}$. By comparing the mean lasing threshold from each sample using ANOVA, we found nanowires transferred by a $5 \mathrm{~s}$ ultrasound bath and PDMS to have a statisticallysignificant lower threshold and a lasing yield of $\sim 72 \%$ and $60 \%$ respectively. While a significant variation in length was 
observed between these two samples, we attribute threshold gain reduction from samples transferred by PDMS to the improvement in end-facet quality at the base of nanowires as confirmed by SEM. For this reason, we suggest that PDMS stamp-transfer is the preferred method when single nanowire laser devices are required, and particularly where nanowire selection is possible. ${ }^{28}$ We note that while this study reflects the effect of different transfer methods on nanowire performance for a specific architecture, the large scale optical technique here used can be extended to a wide range of applications for nanowire characterization. We thus demonstrate a new robust methodology for understanding fabrication processing for bottom up nanotechnology towards high yield nanolasing.

\section{Conflicts of interest}

There are no conflicts to declare.

\section{Acknowledgements}

We thank the Australian National Fabrication Facility (ANFF) ACT node for access to the epitaxial facility used in this work. Additionally, we thank Jose Ignacio Cuitún from the Cathie Marsh Institute for Social Research at the University of Manchester for his assistance on ANOVA and Tukey-Kramer statistical analysis. PP acknowledges the support of the Royal Society (RG140411). JAA acknowledges a CONACyT-SENER funded scholarship. TB, ML, HHT and CJ acknowledge financial support from the Australian Research Council. This work was supported by the Henry Royce Institute for Advanced Materials, funded through EPSRC grants EP/R00661X/1, EP/P025021/1 and $\mathrm{EP} / \mathrm{P025498/1.}$

\section{Notes and references}

1 P. Yang, R. Yan and M. Fardy, Nano Lett., 2010, 10, 15291536.

2 H. J. Joyce, Q. Gao, H. Hoe Tan, C. Jagadish, Y. Kim, J. Zou, L. M. Smith, H. E. Jackson, J. M. Yarrison-Rice, P. Parkinson and M. B. Johnston, Prog. Quantum Electron., 2011, 35, 23-75.

3 N. P. Dasgupta, J. Sun, C. Liu, S. Brittman, S. C. Andrews, J. Lim, H. Gao, R. Yan and P. Yang, Adv. Mater., 2014, 26, 2137-2183.

4 Y. Zhang, G. Davis, H. A. Fonseka, A. Velichko, A. Gustafsson, T. Godde, D. Saxena, M. Aagesen, P. W. Parkinson, J. A. Gott, S. Huo, A. M. Sanchez, D. J. Mowbray and H. Liu, ACS Nano, 2019, 13, 5931-5938.

5 C. Couteau, A. Larrue, C. Wilhelm and C. Soci, Nanophotonics, 2015, 4, 90-107.

6 D. Saxena, S. Mokkapati, P. Parkinson, N. Jiang, Q. Gao, H. H. Tan and C. Jagadish, Nat. Photonics, 2013, 7, 963-968.

7 D. Saxena, N. Jiang, X. Yuan, S. Mokkapati, Y. Guo, H. H. Tan and C. Jagadish, Nano Lett., 2016, 16, 5080-5086.

8 T. Burgess, D. Saxena, S. Mokkapati, Z. Li, C. R. Hall, J. A. Davis, Y. Wang, L. M. Smith, L. Fu, P. Caroff, H. H. Tan and C. Jagadish, Nat. Commun., 2016, 7, 11927.
9 T. Stettner, P. Zimmermann, B. Loitsch, M. Döblinger, A. Regler, B. Mayer, J. Winnerl, S. Matich, H. Riedl, M. Kaniber, G. Abstreiter, G. Koblmüller and J. J. Finley, Appl. Phys. Lett., 2016, 108, 1-5.

10 B. Chen, H. Wu, C. Xin, D. Dai and L. Tong, Nat. Commun., 2017, 8, 20.

11 G. Koblmüller, B. Mayer, T. Stettner, G. Abstreiter and J. J. Finley, Semicond. Sci. Technol., 2017, 32, 053001.

12 T. Stettner, A. Thurn, M. Döblinger, M. O. Hill, J. Bissinger, P. Schmiedeke, S. Matich, T. Kostenbader, D. Ruhstorfer, H. Riedl, M. Kaniber, L. J. Lauhon, J. J. Finley and G. Koblmüller, Nano Lett., 2018, 18, 6292-6300.

13 S. Chen, M. Yukimune, R. Fujiwara, F. Ishikawa, W. M. Chen and I. A. Buyanova, Nano Lett., 2019, 19, 885-890.

14 Y. Zhang, D. Saxena, M. Aagesen and H. Liu, Nanotechnology, 2019, 30, 192002.

15 W.-Z. Xu, F.-F. Ren, D. Jevtics, A. Hurtado, L. Li, Q. Gao, J. Ye, F. Wang, B. Guilhabert, L. Fu, H. Lu, R. Zhang, H. H. Tan, M. D. Dawson and C. Jagadish, Nano Lett., 2018, 18, 34143420.

16 L. Tong, J. Lou, R. R. Gattass, S. He, X. Chen, L. Liu and E. Mazur, Nano Lett., 2005, 5, 259-262.

17 P. Parkinson, H. J. Joyce, Q. Gao, H. H. Tan, X. Zhang, J. Zou, C. Jagadish, L. M. Herz and M. B. Johnston, Nano Lett., 2009, 9, 3349-3353.

18 J. A. Alanis, D. Saxena, S. Mokkapati, N. Jiang, K. Peng, X. Tang, L. Fu, H. H. Tan, C. Jagadish and P. Parkinson, Nano Lett., 2017, 17, 4860-4865.

19 J. A. Alanis, M. Lysevych, T. Burgess, D. Saxena, S. Mokkapati, S. Skalsky, X. Tang, P. Mitchell, A. S. Walton, H. H. Tan, C. Jagadish and P. Parkinson, Nano Lett., 2019, 19, 362-368.

20 B. Sun, M. Fernandez and A. S. Barnard, Nanoscale Horiz., 2016, 1, 89-95.

21 J. G. Mendoza-Alvarez, F. D. Nunes and N. B. Patel, J. Appl. Phys., 1980, 51, 4365-4367.

22 B. Bennett, R. Soref and J. Del Alamo, IEEE J. Quantum Electron., 1990, 26, 113-122.

23 H. L. Chen, C. Himwas, A. Scaccabarozzi, P. Rale, F. Oehler, A. Lemaître, L. Lombez, J. F. Guillemoles, M. Tchernycheva, J. C. Harmand, A. Cattoni and S. Collin, Nano Lett., 2017, 17, 6667-6675.

24 R. Röder and C. Ronning, Semicond. Sci. Technol., 2018, 33, 033001.

25 W. C. Driscoll, Comput. Ind. Eng., 1996, 31, 265-268.

26 T. Nobis and M. Grundmann, Phys. Rev. A: At., Mol., Opt. Phys., 2005, 72, 1-11.

27 Y.-B. Wang, L.-F. Wang, H. J. Joyce, Q. Gao, X.-Z. Liao, Y.-W. Mai, H. H. Tan, J. Zou, S. P. Ringer, H.-J. Gao and C. Jagadish, Adv. Mater., 2011, 23, 1356-1360.

28 A. Hurtado, D. Jevtics, B. Guilhabert, Q. Gao, H. H. Tan, C. Jagadish and M. D. Dawson, Novel nanoscale transfer printing technique for precise positioning of nanowire lasers, SPIE Newsroom, 2017, vol. 10-12, DOI: 10.1117/ 2.1201612.006830. 